\title{
SPECIAL NOTE
}

\section{Circadian rhythms and animal behavior research: A follow-up survey}

\author{
STEPHEN H. HOBBS and STEVEN J. GOLDBERG \\ Augusta College, Augusta, Georgia
}

\begin{abstract}
An updated survey of animal behavior research articles published between 1981 and 1986 was conducted to assess the frequency with which potentially important information about circadian rhythms was specified. Although the percentage of sampled articles that reported circadian details was greater in this survey than in a previous one, research reports are still deficient in providing enough information for researchers to undertake reasonable replications. Furthermore, the practice of conducting behavioral research on animals during their normal sleep, or inactive, phase was found to be characteristic of reports published during the time period covered by the survey.
\end{abstract}

Rhythms in nature have long been recognized, but serious investigations into their impact on behavior have been rather recent. Topics receiving attention today are as diverse as the impact of shift work and time-zone changes on performance, the possible linkage of neurotransmitter rhythmicities to depression or psychosis, the determination of optimal work hours for individuals, and the elucidation of the functions of alternating REM and NREM sleep (see Moore-Ede, Sulzman, \& Fuller, 1982; Webb, 1982). Along with this wave of interest, a number of journals and books devoted solely to biological rhythms have appeared, and many textbooks in psychology presently provide discussion of rhythm-behavior relationships.

Basic animal research on rhythms lasting about $24 \mathrm{~h}$ (circadian rhythms) have been especially fruitful in revealing numerous behavioral fluctuations. Such findings have certain implications for the conduct of psychological research in general. For example, apparent conflicts between results obtained in two different laboratories could be due simply to differences in times in their daily cycles that subjects were tested. Resolution of such conflicts could come from testing subjects at more than one point in a rhythm's cycle or by at least stating when subjects were tested, so that post hoc comparisons across laboratories could be made.

Hobbs (1981) conducted a survey of animal behavior research to determine the extent to which investigators did report rhythm-relevant information, thereby facilitating such comparisons. After reviewing a sample of the published animal behavior research in the 5-year period between 1976 and 1980, he concluded that so few details

Reprint requests should be sent to Stephen H. Hobbs, Department of Psychology, Augusta College, 2500 Walton Way, Augusta, GA 30910. about rhythms were included that realistic comparisons, and especially replications, would be nearly impossible. This led to the suggestion that information about biological rhythms, such as maintenance light-dark (LD) cycles, "should be reported as routinely as subject age, gender, and species"' (p. 604). The present study was undertaken (1) to determine if changes in the reporting of rhythm information in published research had occurred since the time of the initial survey, (2) to ascertain any trends within the last decade in research publication of circadian rhythms and behavior, and (3) to learn if journals have editorial policies about the inclusion of biological rhythm information in their published articles.

\section{METHOD}

Except as noted below, the procedures used by Hobbs (1981) were repeated as closely as possible. Approximately $10 \%$ of the research articles published in each of five animal behavior journals over the years 1981-1986 were reviewed. Due to the comparatively much greater number of articles published per year in Physiology \& Behavior, only $3 \%$ of its articles were selected. The remaining journals surveyed were Animal Learning \& Behavior, Journal of Comparative and Physiological Psychology (only Behavioral Neuroscience was included after the journal division), Journal of Experimental Psychology: Animal Behavior Processes, and Physiological Psychology (now Psychobiology). Experimental investigations were selected randomly with the restrictions that no author be included more than once each year and that no human studies be included. If an article reported more than one experiment, only the first was considered. The total number of articles in the survey sample was 207.

Method sections were examined to determine if the article specified the maintenance LD cycles of the experimental subjects, the time of testing, and the lighting conditions during testing. Details about these items, if provided, were recorded along with the species of animal used. Finally, it was noted whether a study had circadian or other biological rhythms as a focal point of the investigation. 
To further assess the frequency with which research on this topic area was being published in the psychological literature, a computer count from the Psychological Abstracts database for the years 1976-1986 was made using the phrase "animal circadian rhythms." A manual count using the Psychological Abstracts index during the same interval was made additionally as a complement.

In the summer of 1986 , a letter was sent to the editor of each of the surveyed journals to inquire about any editorial policy regarding the inclusion of biological rhythm information in published research. The editors were asked to describe any such policy and were invited to share their perspectives on the issues raised.

\section{RESULTS AND DISCUSSION}

Only $10 \%$ of the studies in the sample provided information on all three items of interest. Table 1 presents the separate frequency data for these three items, along with the percentages obtained in the earlier survey (Hobbs, 1981). Although the frequency with which authors reported test lighting conditions had doubled, only small or moderate increases for reporting other rhythm-relevant information had occurred. As with the initial survey, variability remained high: subsamples by year and journal ranged from providing no target content to providing high information frequency.

It would seem logical that reporting one item would appreciably increase the likelihood of specifying other rhythm-relevant information. As can be seen from Table 2 , this did not always occur. For example, even when authors reported their animals' housing cycle, there was less than a 50:50 chance that time of testing within that cycle would be conveyed.

Percentages of studies using various LD cycles, when that information was provided, is presented in Table 3. Summing over the first four categories shows that approximately four-fifths of the studies that reported LD cycles employed a schedule in which lights were on during nor-

Table 1

Percentage of Studies Reporting Item Information

\begin{tabular}{|c|c|c|c|c|}
\hline \multirow[b]{3}{*}{ Item } & \multicolumn{4}{|c|}{ Survey Period } \\
\hline & \multicolumn{2}{|c|}{$1976-1980$} & \multicolumn{2}{|c|}{ 1981-1986 } \\
\hline & Mean & Low/High & Mean & Low/High \\
\hline Housing Cycle & 50 & $0 / 90$ & 62 & $0 / 100$ \\
\hline Testing Time & 26 & $0 / 67$ & 28 & $0 / 67$ \\
\hline Test Lighting & 21 & $0 / 80$ & 42 & $0 / 100$ \\
\hline
\end{tabular}

Note-Low/high columns refer to the lowest and highest percentages from any journal, any year.

Table 2

Contingency Table of Percentages of Studies Reporting Item Information Given That Other Information Was Also Reported Then

If Housing Cycle Testing Time Test Lighting

\begin{tabular}{lccc}
\hline Housing Cycle (1) & - & 40 & 40 \\
Testing Time (2) & 86 & - & 45 \\
Test Lighting (3) & 59 & 32 & - \\
Both (1) and (2) & - & - & 50 \\
Both (1) and (3) & - & 52 & - \\
Both (2) and (3) & 96 & - & - \\
\hline
\end{tabular}

Table 3

Percentage Studies Employing Different LD Cycles

\begin{tabular}{lcc}
\hline & \multicolumn{2}{c}{ Survey Period } \\
\cline { 2 - 3 } Type & $1976-1980$ & $1981-1986$ \\
\hline $\begin{array}{l}\text { 12:12 L/D } \\
\text { (lights on in a.m.) }\end{array}$ & 56 & 59 \\
14:10 L/D & 10 & 12 \\
(lights on in a.m.) & & \\
16:8 L/D & 4 & 8 \\
(lights on in a.m.) & & \\
Natural Illumination & 1 & 3 \\
Reversed L/D & 11 & 12 \\
Continuous Illumination & 9 & 0 \\
Other & 9 & 6 \\
\hline
\end{tabular}

mal active hours for humans. Since $77 \%$ of the sampled studies used nocturnal rodents, and since we have little information available to the contrary, it appears that a considerable amount of animal behavior research is being conducted during subjects' normal periods of sleep or inactivity. Indeed, in those studies using nocturnal animals where both LD cycles and time of testing are specified, the animals were tested outside of their normal activity phase fully $72 \%$ of the time.

Approximately $2 \%$ of the articles both in the present survey and in the earlier survey were specifically investigating circadian or biological rhythms. Similarly, the computer and manual counts of articles dealing with animal circadian rhythms showed no clear trend, with an average of 77 articles per year being fairly representative of the past decade.

All journal editors replied to the letter of inquiry. Each indicated that no formal policy regarding the inclusion of biological rhythm information in method sections was currently being used. Comments ranged from suggesting that competent peer reviewing is sufficient to address the issue to asserting that a formal editorial policy would be established. Such a policy was recently announced by the editors of one of the surveyed journals (Nadel, Barnes, Gallagher, Kolb, \& Willner, 1987).

A survey of animal behavior researchers themselves might reveal considerable awareness of the potential impact circadian rhythms can have on animal behavior. However, results of the current study would suggest that this knowledge may not be reflected in the actual conduct of animal behavior research, or at least in the reporting of that research in published form. On the other hand, more rhythm-relevant information was provided in recent research reports than had been provided in earlier ones, and at least one psychology journal has established an editorial policy addressing the issue.

As Terman (1983) has pointed out, the psychologist who has research interests focused elsewhere may regard circadian variation primarily as a confounding factor that should be eliminated. Since it is unlikely that ignoring rhythmicity will be very beneficial, some minimal attention to rhythms appears in order. Terman provided an ex- 
cellent discussion of ways in which experimental design could better reflect our current knowledge about circadian rhythms (see also, Webb, 1982). Although laboratory limitations and other considerations may prevent researchers from attaining the "ideal," a general acceptance of complete reporting of known rhythm-relevant information would be an important beginning.

\section{REFERENCES}

Hoвbs, S. H. (1981). Circadian rhythms and animal behavior research. Animal Learning \& Behavior, 9, 604-605.
Moore-Ede, M. C., Sulzman, F. M., \& Fuller, C. A. (1982). The clocks that time us. Cambridge, MA: Harvard University Press.

Nadel, L., Barnes, C., Gallagher, M., Kolb, B., \& Willner, J. (1987). Editorial. Psychobiology, 15, 1.

Terman, M. (1983). Behavioral analysis and circadian rhythms. In M. D. Zeiler \& P. Harzen (Eds.), Biological factors in learning (pp. 103-141). New York: Wiley.

WEBB, W. B. (1982). Sleep, biological rhythms, performance research: An introduction. In W. B. Webb (Ed.), Biological rhythms, sleep, and performance (pp. 1-25). New York: Wiley.

(Manuscript received May 26, 1987; revision accepted for publication July $15,1987$. 